\title{
Letter regarding "Prognostic role of cytovillin expression in patients with osteosarcoma: a meta-analysis" by Guo et al.
}

\author{
Peng Wang • Lei Sun • Lei Yu • Hu Wang
}

Received: 26 December 2013 / Accepted: 22 January 2014 / Published online: 8 February 2014

(C) International Society of Oncology and BioMarkers (ISOBM) 2014

To the Editor,

Guo et al. [1] conducted a meta-analysis to evaluate the role of cytovillin expression on the overall survival rate by calculating the pooled risk ratio (RR) with the corresponding $95 \%$ confidence interval $(95 \% \mathrm{CI})$. They concluded that the cytovillin expression is obviously associated with lower overall survival rate in osteosarcoma patients, and it is an effective biomarker of prognosis. Before accepting their conclusions, we would like to express some concerns in relation to their meta-analysis.

To begin with, the investigators did not provide us with the flow diagram of the study selection procedure. To make us read the meta-analysis better, the investigators should have provided one.

Furthermore, we suggest that the investigators should evaluate the methodological quality of the selected studies, which

\section{P. Wang}

Department of Orthopedics, The Fourth Hospital of Harbin Medical University, Harbin 150001, Heilongjiang Province, People's

Republic of China

\section{Sun}

Department of Ophthalmology, The Fourth Hospital of Harbin Medical University, Harbin 150001, Heilongjiang Province, People's Republic of China

\section{Yu}

Department of Infectious Disease, The Fourth Hospital of Harbin Medical University, Harbin 150001, Heilongjiang Province, People's Republic of China

\section{H. Wang $(\square)$}

Department of Orthopedics, First Affiliated Hospital of Shantou University Medical College, Shantou 515041, Guangdong Province, People's Republic of China

e-mail: wangh041@163.com could avoid the potential bias in the meta-analysis. Each included paper could be independently assessed by two investigators using a standardized electronic form of predefined criteria. Meanwhile, the investigators could describe how to evaluate the quality of all studies in the meta-analysis.

In addition, the investigators included eight studies including 415 osteosarcoma patients. However, the investigators did not provide us any characteristics of these eligible studies. For us to read the meta-analysis well, we suggest that the investigators should provide us with the characteristics, such as patient source, histo-subtype (Osteo\%), stage of osteosarcoma, RR estimation, and survival results.

Eventually, in the "Results" section, the investigators used a forest plot to show the effect of cytovillin expression on the overall survival rate. However, we are wondering what does the overall survival rate, 2-year survival, or 5-year survival mean? Meanwhile, the investigators did not evaluate the effect of cytovillin expression on clinical stage and the neoplasm metastasis. In our opinion, these should be added in the "Results" section to make a better meta-analysis.

In a word, more studies with large sample size are needed to provide a more comprehensive evaluation of the prognostic role of cytovillin expression in patients with osteosarcoma.

Conflicts of interest None.

\section{Reference}

1. Guo SB, Bai R, Zhao W, Wang YX, Zhao ZQ, Feng W. Prognostic role of cytovillin expression in patients with osteosarcoma: a metaanalysis. Tumor Biol. 2013. doi:10.1007/s13277-013-1065-8. 